\title{
The sentinel node in pelvic gynaecological tumors: an updated view
}

\begin{abstract}
Summary
The sentinel lymph node (SLN) refers to the first lymph node susceptible to being affected, due to the lymphatic drainage of a primary tumor. The lymph node involvement in cancer patients is one of the main prognostic factors. Without affecting the prognosis at any time, the SLN seeks to reduce the morbidity associated with lymphadenectomy, reducing surgical time, reducing intraoperative and postoperative complications, such as lymphedema or neuralgia and costs associated with conventional surgery. Although its use has extensive experience in tumors such as breast, the SG in gynecological tumors is still in the early stages. With this review we intend to make a close and current view of the use of this technique in malignant tumors of the female genital tract.
\end{abstract}

Keywords: sentinel lymph node, gynecological tumors, indocyanine green, technetium-99m, blue dye, fluorescent real-time mapping
Volume 10 Issue 3 - 2019

\author{
Duro Gómez Jorge,' Zuheros Montes José \\ David,' Rodríguez Marín Ana Belén, ${ }^{2}$ Nieto \\ Espinar Yolanda, ${ }^{3}$ Castelo-Branco Camil ${ }^{4}$ \\ 'Department of Gynecology and Obstetrics, Reina Sofía \\ University Hospital, Spain \\ ${ }^{2}$ Department of Gynecology and Obstetrics, Hospital San Juan \\ de Dios, Spain \\ ${ }^{3}$ Department of Gynecology and Obstetrics, Hospital \\ Universitario San Agustín, Spain \\ ${ }^{4}$ Clinic Institute of Gynecology, Obstetrics and Neonatology- \\ Hospital Clinic, Faculty of Medicine-University of Barcelona, \\ Institut d'Investigacions Biomèdiques August Pi i Sunyer \\ (IDIBAPS), Spain
} Gynecology, Obstetrics and Neonatology, Hospital Clinic Villarroel I70, 08036-Barcelona, Spain, Tel + 34932275436 Fax + 399322793 25, Email castelobranco@ub.edu

Received: May 28, 2019 | Published: July 08, 2019

\section{Introduction}

The sentinel lymph node (SLN) refers to the first lymph node susceptible to being affected, due to the lymphatic drainage of a primary tumor. ${ }^{1}$ Initially, it was implanted for penile cancer ${ }^{2}$ followed by melanoma and breast cancer. ${ }^{3}$ The lymph node involvement in cancer patients is one of the main prognostic factors.

Regarding pelvic gynecological tumors, the first cancer in which it began to be implemented was for the vulva and has spread to cervical or endometrial cancer. Knowing the affectation or no ganglion besides not only will mark the prognosis ${ }^{4}$ determines, but also the adjuvant treatment in these patients. In addition, selective SLN biopsy is considered the strongest predictor of distant metastasis, particularly when its affectation is evaluated by immunohistochemistry with antibodies against the factor VIII or CD31-related antigen. ${ }^{5}$ Thanks to this, we will be in the habit of carrying out an adequate therapeutic strategy.

The implementation of SLN biopsy has among its objectives the reduction of morbidity associated with lymphadenectomy, ${ }^{6}$ the reduction of surgical time, the reduction of intraoperative ${ }^{7}$ and postoperative complications, such as lymphedema or neuralgia, ${ }^{8}$ as well as reducing the costs associated with conventional surgery. All this must be done without entailing a worsening of the prognosis of the target disease.

Although this technique has been accepted as an alternative to pelvic and para-aortic lymphadenectomy, ${ }^{9}, 10$ it can be difficult to expose and understand by our patients, especially in cases in which it is associated with an increased risk of recurrence. ${ }^{11}$ Perhaps these patients are not willing to assume a higher risk at the expense of reducing the radicality of surgery, something that physicians are. It is a challenge on the part of the different specialists, to try to improve the results so that SLN does not reduce the treatment of oncological patients. For this, it will be essential to make an adequate selection of patients. Thus, we will increase our detection capacity in patients with early staging and lower tumor sizes. ${ }^{5}$ Before completely replacing the lymphadenectomy, ongoing controlled trials should explore and confirm the additional value of SLN biopsy in both perioperative morbidity and survival. ${ }^{12}$ In this review, we intend to perform an update on the status of the sentinel node within the current gynecology.

\section{Indications}

In order to a certain tumor to be susceptible to SLN biopsy, it must follow a consecutive lymphatic drainage through a certain lymph node chain. In addition, there must be absence of disease at a distance and lymph node involvement both at the clinical level and in the imaging tests. ${ }^{13}$ Also, as a rule the disease should not be locally advanced.

In this way, SLN biopsy, whether by laparoscopy, laparotomy or robotic surgery, has proven to be feasible, efficient, safe and imitable. ${ }^{13}$ This technique SLN biopsy has been shown to be safe and feasible in several gynecological cancers such as vulvar cancer, cervical cancer and endometrial cancer.

\section{Detection methods}

There are different tracers used for the detection of SLN. Among them is technetium 99 (Tc99) or methylene blue (MB), with a detection of $66 \%$ to $86 \%$. The most recent appearance is the use of 
fluorescent matrices such as indocyanine green (ICG), whose use is increasing within the different protocols. ${ }^{14}$ ICG together with the near infrared fluorescence has gained a great utility with the advantage of providing real-time images during surgery. It is experimental, the use of carbon nanoparticles for labeling remains. ${ }^{15}$

The superiority of MB has been shown together with the tracer versus MB only. ${ }^{16,17}$ However, ICG has shown greater detection capacity than MB and Tc $99^{\mathrm{m}} .{ }^{18}$ In this prospective study by Holloway et al., ${ }^{19}$ the ICG detected more SLN and more metastases than the MB and without safety problems. ${ }^{19}$ ICG has an excellent toxicity profile, with higher overall and bilateral detection rates compared to MB and higher bilateral detection rates compared to a combination of Tc-99m and blue dye. ${ }^{20,21}$ There are available studies that even underestimate the use of MB in favor of Tc99 or ICG. ${ }^{22}$ Given this, whenever possible, the use of ICG should be favored over the other tracers for SLN biopsy. ${ }^{23}$

One of the variables to consider, is the time elapsed from the injection of the tracer until its detection. This period is related to the possible failure of intraoperative detection. Kushner et al. reported that the best time to detect sentinel lymph nodes with the MB was 30 minutes after the dye injection. 50 minutes after the injection the ICG could not be identified. ${ }^{24}$ On the other hand, the ICG presents a good detection, from 5 to 60 minutes after its injection. ${ }^{25}$ Taking into consideration the time in which the ICG should be detected, its realtime detection achieves better results with a higher bilateral detection rate. ${ }^{26}$ In addition to the time elapsed, it is important to perform the injection of the tracer properly. There are different modalities depending on the type of tumor..$^{27,28}$

In addition to those mentioned, there are other factors that may affect the detection rate of the SLN and should be considered within the next protocols and studies. Among them and to highlight, the detrimental effect of body mass index (BMI) on ICG detection rates as a marker in obese patients. ${ }^{29}$

\section{Vulva}

An adequate diagnostic and therapeutic strategy in the approach to vulvar cancer is essential due to survival is better when the treatment is rapidly established after diagnosis. The involvement of the inguinal or femoral nodes is the main prognostic factor. ${ }^{30}$ In this article, we review the published data supporting the SLN biopsy as part of the standard treatment for women with early-stage vulvar cancer and discuss future considerations for the treatment of this disease. ${ }^{31}$

Adequate selection of patients with squamous cell carcinoma of the vulva is essential. ${ }^{32}$ The SLN biopsy in vulvar cancer should be limited to stages IB and II of the FIGO which they are unifocal tumors, less than $4 \mathrm{~cm}$ and with clinical and radiological absence of lymph node involvement. This way, without affecting the prognosis, we achieved a reduction in operative mortality. ${ }^{33}$ In 2008, the first Groningen international study on sentinel lymph nodes in vulvar cancer (GROINSS-V) demonstrated that the omission of inguino-femoral lymphadenectomy is safe in patients with early-stage vulvar cancer and negative SLN, simultaneously decreasing morbidity related to the treatment. There are sufficient studies that corroborate the viability, safety and reproducibility of SLN biopsy in these tumors. ${ }^{34}$ Some of them, such as Slomovitz, show how SLN biopsy is associated with a better quality of life than complete lymphadenectomy, is more cost-effective than complete lymphadenectomy and achieves a better pathological evaluation. ${ }^{32}$
Something to highlight in the SLN biopsy in vulvar tumors is the ultrastaging, since the introduction of this procedure in a standardized way, more and lower inguinofemoral ganglion metastases have been diagnosed. The true clinical impact of micrometastases is unknown. What seems clear is that the larger size of the SLN metastases, make greater the chances of metastasis in non-sentinel lymph nodes and the lower survival rates. In this way, the size of metastases to the lymph nodes is included in the last staging system for vulvar cancer. However, micrometastases have not been included in the staging of this type of tumors. Further studies are needed to determine the clinical consequences of the size of the SLN metastases. ${ }^{35}$

From a practical point of view, we have extensive experience in the use of technetium as a tracer. However, there are already studies that have shown that indocyanine green has a similar sensitivity, so it should be evaluated in new protocols in order to can incorporate it into our usual practice. ${ }^{36}$

In short, we can see how it has been shown that complete inguinofemoral lymphadenectomy is no longer necessary in most patients with this disease and there are no excuses for not incorporating SLN biopsy in the approach to vulvar cancer.

\section{Cervix}

Cervical cancer is one of the most frequent gynecological tumors in young patients, being one of the main causes of death by this reason in developed countries. Frequently, at diagnosis the tumor is limited to the cervix, around $47 \%$ according to OonK. ${ }^{35}$ The usual approach of these patients goes through radical hysterectomy or trachelectomy with bilateral pelvic lymphadenectomy. ${ }^{1,37}$ This has achieved a high survival at 5 years when it comes to early stages. ${ }^{38,39}$ The SLN biopsy is an appropriate option in this type of patients. ${ }^{40}$

The success of SLN biopsy in cervical cancer will be based on an adequate selection of patients in which the tumor is small and is treated with low staging. In general, it should be limited to stages IA2 and IBI. The best detection rate is found in tumors below $2 \mathrm{~cm} .{ }^{41}$ However, it should be considered that currently, it is an experimental technique with a low implantation rate..$^{42}$

To perform SLN biopsy in cervical cancer, MB can be used with or without Tc99. It must be injected into the cervix immediately after the anesthesia had been established. ${ }^{41}$ This can be established in each quadrant of the cervix, either at 3 and 9 o'clock in the cervix. Intraoperatively, $\mathrm{MB}$ an be located at a glance.

There are studies that compare these markers with indocyanine green. In patients with early-stage cervical cancer, a higher bilateral detection was confirmed using the IG instead of standard techniques. ${ }^{43}$ It was also shown in this study by Buda et al. where the conization did not have a significant impact on the detection rate of the lymph nodes either with MB+Tc99 or with ICG. In the case of advanced cervical cancer (stage IB $1>2 \mathrm{~cm}$ ), the detection rate was higher with ICG than with $\mathrm{MB}+\mathrm{Tc} 99$.

So that we can see that ICG is a promising tool for SLN in cervical cancer, since it seems less affected by the stage of the disease, with a higher detection rate compared to traditional methods. ${ }^{42}$ In addition, currently the detection of the sentinel node with GI by means of robotics with the da Vinci Xi supposes an added advantage. ${ }^{44}$

We want to highlight an additional advantage of SLN biopsy in cervical cancer. This is the possibility of detecting areas outside of 
what includes the usual lymphadenectomy, thus being able to have additional histological information. On the contrary, there are some scenarios that make more studies necessary, such as its application in conservative fertility surgery and in patients with neoadjuvant chemotherapy. ${ }^{45}$ In any case, the superiority of SLN biopsy over PET in the detection of lymph node disease has been demonstrated. ${ }^{46,47}$

Although it is a new technique that will require more studies, according to the National Comprehensive Cancer Network guidelines, the BSGC is a viable option for the management of this type of tumors, including it within its therapeutic guidelines. ${ }^{23,30} \mathrm{In}$ conclusion, according to the available evidence, the BSCG is a safe procedure with high detection rate and low false negative rate. ${ }^{44}$

\section{Endometrium}

Endometrial cancer is the most frequent cancer of the female genital tract in the US with 6,180 cases in $2017 .{ }^{36}$ So, if there is a gynecological cancer where the implementation of SLN biopsy is most interesting, it is in this tumor. In patients in initial stages, it is very important to perform a systematic screening of patients without metastasis to lymph nodes to reduce the range of surgical resection, and to reduce the incidence of the corresponding complications and improve the quality of life of the patients. ${ }^{14}$ SLN biopsy allows the reduction of unnecessary lymphadenectomies and reduces the risk of underdiagnosis in patients with metastatic lymph nodes. ${ }^{48}$ However, this technique has the difficulty of the double lymphatic drainage pathway of these tumors. ${ }^{49}$ In addition, the different existing types must be considered. ${ }^{50,51}$ According to the FIRES study, the SLN biopsy has a sensitivity of $97.2 \%$ and a negative predictive value of $99.6 \% .{ }^{51}$

As in previous cases, several protocols can be used to the SLN biopsy in endometrial cancer. Among them, the Tc 99, the MB or the ICG. ICG has an excellent toxicity profile, with higher overall and bilateral detection rates compared to blue dyes, as well as higher bilateral detection rates compared to a combination of Tc-99m and MB. ${ }^{26,50,51}$ The ICG in addition to an excellent detection rate shows a low rate of false negatives This superiority has been revealed even when we speak of advanced stages (IIIC) ${ }^{51}$ or high risk types, achieving a sensitivity, rate of FN and VPN acceptable. In this way, the SLN biopsy with ICG has managed to reduce the total number of complete lymphadenectomies, reducing the duration and the additional costs of surgical treatment. ${ }^{52-55}$

The study published by Niikura shows how the tracer injection in the cervix is very sensitive in the detection of SLN metastases in early stage endometrial cancer (stages I and II of FIGO). This is a particularly useful and safe modality when combined with injection of blue dye into the uterine body. ${ }^{56}$ Controversy exists on the place of injection of the tracer. In this regard, the study published by Rossi shows how cervical ICG injection achieves a higher rate of SLN detection and an anatomical lymph node distribution similar to hysteroscopic endometrial injection in patients with endometrial cancer. ${ }^{57}$

In summary, BSCG with GI is a feasible technique with high diagnostic precision that could eventually displace conventional lymphadenectomy in the coming years, thus reducing the associated morbidity and mortality. ${ }^{51}$

\section{Ovary}

There are few studies about SLN biopsy in ovarian cancer. Anatomy, various histological types and their drainage, or tracer injection, are some of the difficulties that contribute to the limited scientific evidence available. An example of this is the study of the Buddha where ICG is used for aortic staging in 10 patients with ovarian cancer. In 9 cases the SLN was detected in the aortic region after the injection of the tracer. In 3 cases, SLN were also identified in the common iliac region. ${ }^{58}$

\section{Costs}

There are few studies that evaluate the costs associated with the use of the sentinel lymph node. A sample of this is the study of Buddha, who showed that with SLN biopsy in endometrial cancer there is a reduction of more than 1000 dollars compared to conventional lymphadenectomy. Assuming a total of 66 million dollars less for the total cases of ca. Endometrium in the USA. On the other hand, when we refer to vulvar cancer, SLN biopsy seems the least expensive alternative, mainly due to the great impact of lymphedema associated with conventional surgery on quality of life. ${ }^{59}$ According to the study of van der Vorst, with the SLN biopsy in vulvar cancer a reduction of 22416 dollars on an annual basis with respect to conventional surgery. ${ }^{60}$

When comparing the use of the different tracers, the option that the ICG uses seems to be the most profitable strategy. ${ }^{61,62}$

\section{Conclusion}

The BSGC in gynecological tumors is a current technique that manages to reduce the morbidity and mortality associated with traditional surgery, in addition to achieving a reduction in costs associated with it. This is also achieved, without interfering in the prognosis of these patients. New standardized studies are needed to reinforce and homogenize this technique so that we can incorporate it into our usual protocols.

\section{Acknowledgments}

None.

\section{Conflicts of interest}

The authors report no declarations of interest.

\section{References}

1. Holman LL, Levenback CF, Frumovitz M. Sentinel lymph node evaluation in women with cervical cancer. $J$ Minim Invasive Gynecol. 2014;21(4):540-545.

2. Cabanas RM. An approach for the treatment of penile carcinoma. Cancer. 1977;39(2):456-466.

3. Lècuru F, Mathevet P, Querleu D, et al. Bilateral negative sentinel nodes accurately predict absence of lymph node metástasis in early cervical cancer: Results of the SENTICOL study. J Clin Oncol. 2011;29(13):16861691.

4. Fuller AF, Elliott N, Kosloff C, et al. Determinants of increased risk for recurrence in patients undergoing radical hysterectomy for stage IB and IIA carcinoma of the cervix. Gynecol Oncol. 1989;33(1):34-39.

5. Lax SF, Tamussino KF, Lang PF. Metastatic mechanisms of uterine malignancies and therapeutic consequences. Pathologe. 2016;37(6):549-556.

6. Cea García J, de la Riva Pérez PA, Rodríguez Jiménez I, et al. 2018. Selective biopsy of the sentinel node in cancer of cervix: Experience in validation phase. Rev Esp Med Nucl Imagen Mol. 2018;37(6):359-365. 
7. Matsuura Y, Kawagoe T, Toki N, et al. Long-standing complications after treatment for cancer of the uterine cervix--clinical significance of medical examination at 5 years after treatment. Int $J$ Gynecol Cancer. 2006;16(1):294-297.

8. Wisner KPA, Ahmad S, Holloway RW. Indications and techniques for robotic pelvic and para-aortic lymphadenectomy with sentinel lymph node mapping in gynecologic oncology. Best Pract Res Clin Obstet Gynaecol. 2017;45:83-93.

9. Koh WH, Greer BE, Abu-Rustum NR, et al. Cervical cancer, version 2. J Natl Compr Canc Netw. 2015;13(4):395-404.

10. Gold MA, Tian C, Whitney CW, et al. Surgical versus radiographic determination of para-aortic lymph node metastases before chemoradiation for locally advanced cervical carcinoma. Cancer. 2008;112(9):19541963.

11. Gungorduk K, Kocian R, Basaran D, et al. Are patients and physicians willing to accept less-radical procedures for cervical cancer? J Gynecol Oncol. 2018;29(4):e50.

12. Buda A, Crivellaro C, Elisei F, et al. Impact of indocyanine green for sentinel lymph node mapping in early stage endometrial and cervical cancer: comparison with conventional radiotracer $(99 \mathrm{~m}) \mathrm{Tc}$ and/ or blue dye. Ann Surg Oncol. 2016;23(7):2183-2191.

13. Lyman GH, Somerfield MR, Bosserman LD, et al. Sentinel lymph node biopsy for patients with early-stage breast cancer: American Society of Clinical Oncology clinical practice guideline update. J Clin Oncol. 2014;32(13):1365-1383.

14. Darin MC, Gómez-Hidalgo NR, Westin SN, et al. Role of Indocyanine green in sentinel node mapping in gynecologic cancer: is fluorescence imaging the new standard?. J Minim Invasive Gynecol. 2016;23(2):186193.

15. Liu KJ, Lv XW, Liu Q, et al. Application of carbon nanoparticles in the laparoscopic sentinel lymph node detection in patients with cervical cancer. Zhongguo Yi Xue Ke Xue Yuan Xue Bao. 2013;35(2):150-154.

16. Hassanzade M, Attaran M, Treglia G, et al. Lymphatic mapping and sentinel node biopsy in squamous cell carcinoma of the vulva: systematic review and meta-analysis of the literature. Gynecol Oncol. 2013;130(1):237-245.

17. Ansari M, Ghodsi-Rad MA, Hassanzadeh M, et al. Sentinel node biopsy in endometrial cancer: systematic review and meta-analysis of the literature. Eur J Gynaecol Oncol. 2013;34(5):387-401.

18. Ruscito I, Gasparri ML, Braicu EI, et al. Sentinel node mapping in cervical and endometrial cancer: indocyanine green versus other conventional dyes-a meta-analysis. Ann Surg Oncol. 2016;23(11):3749-3756.

19. Holloway RW, Ahmad S, Kendrick JE, et al. A prospective cohort study comparing colorimetric and fluorescent imaging for sentinel lymph node mapping in endometrial cancer. Ann Surg Oncol. 2017;24(7):19721979.

20. Papadia A, Zapardiel I, Bussi B, et al. Sentinel lymph node mapping in patients with stage I endometrial carcinoma: a focus on bilateral mapping identification by comparing radiotracer Tc99m with blue dye versus indocyanine green fluorescent dye. J Cancer Res Clin Oncol. 2017;143(3):475-480.

21. Buda A, Papadia A, Zapardiel I, et al. From conventional radiotracer Tc99(m) with blue dye to indocyanine green fluorescence: a comparison of methods towards optimization of sentinel lymph node mapping in early stage cervical cancer for a laparoscopic approach. Ann Surg Oncol. 2016;23(9):2959-2565.

22. How J, Gotlieb WH, Press JZ, et al. Comparing indocyanine green, technetium, and blue dye for sentinel lymph node mapping inendometrial cancer. Gynecol Oncol. 2015;137(3):436-442.
23. Papadia A, Gasparri ML, Buda A, et al. Sentinel lymph node mapping in endometrial cancer: comparison of fluorescence dye with traditional radiocolloid and blue. J Cancer Res Clin Oncol. 2017;143(10):20392048.

24. Kushner DM, Connor JP, Wilson MA, et al. Laparoscopic sentinel lymph node mapping for cervix cancer-a detailed evaluation and time analysis. Gynecol Oncol. 2007;106(3):507-512.

25. Choi HJ, Kim TJ, Lee YY, et al. Time-lapse imaging of sentinel lymph node using indocyanine green with near-infrared fluorescence imaging in early endometrial cancer. J Gynecol Oncol. 2016;27(3):e27.

26. Papadia A, Gasparri ML, Siegenthaler F, et al. FIGO stage IIIC endometrial cancer identification among patients with complex atypical hyperplasia, grade 1 and 2 endometrioid endometrial cancer: laparoscopic indocyanine green sentinel lymph node mapping versus frozen section of the uterus, why get around the problem?. J Cancer Res Clin Oncol. 2017;143(3):491-497.

27. Lantzsch T, Wolters M, Grimm J, et al. Sentinel node procedure in $\mathrm{Ib}$ cervical cancer: a preliminary series. Br J Cancer. 2001;85(6):791-794.

28. Yuan SH, Xiong Y, Wei M, et al. Sentinel lymph node detection using methylene blue in patients with early stage cervical cancer. Gynecol Oncol. 2007;106(1):147-152.

29. Tanaka T, Sasaki S, Tsuchihashi H, et al. 2018. Which is better for predicting pelvic lymph node metastases in patients with cervical cancer: Fluorodeoxyglucose-positron emission tomography/computed tomography or a sentinel nodebiopsy? A retrospective observational study. Medicine. 2018;97(16):e0410.

30. Alkatout I, Schubert M, Garbrecht N, et al. Vulvar cancer: epidemiology, clinical presentation, and management options. Int $J$ Womens Health. 2015;7:305-313.

31. Slomovitz BM, Coleman RL, Oonk MH, et al. Update on sentinel lymph node biopsy for early-stage vulvar cancer. Gynecol Oncol. 2015;138(2):472-477.

32. Levenback CF, Ali S, Coleman RL, et al. Lymphatic mapping and sentinel lymph node biopsy in women with squamous cell carcinoma of the vulva: a gynecologic oncology group study. J Clin Oncol. 2012;30(31):37863791 .

33. Brincat MR, Muscat Baron Y. Sentinel lymph node biopsy in the management of vulvar carcinoma: an evidence-based insight. Int $J$ Gynecol Cancer. 2017;27(8):1769-1773.

34. Van der Zee AG, Oonk MH, De Hullu JA, et al. Sentinel node dissection is safe in the treatment of early-stage vulvar cancer. J Clin Oncol. 2008;26(6):884-889.

35. Oonk MH, Hollema H, van der Zee AG. Sentinel node biopsy in vulvar cancer: implications for staging. Best Pract Res Clin Obstet Gynaecol. 2015;29(6):812-821.

36. Siegel RL, Miller KD, Jemal A. Cancer statistics, 2017. CA Cancer J Clin. 2017;67(1):7-30.

37. Querleu D, Morrow CP. Classification of radical hysterectomy. Lancet Oncol. 2008;9(3):297-303.

38. Nanthamongkolkul K, Hanprasertpong J. Longer waiting times for early stage cervical cancer patients undergoing radical hysterectomy are associated with diminished long-term overall survival. J Gynecol Oncol. 2015;26(4):262-269.

39. Elit L, Fyles AW, Devries MC, et al. Follow-up for women after treatment for cervical cancer: a systematic review. Gynecol Oncol. 2009;114(3):528535 . 
40. Diab Y. Sentinel lymph nodes mapping in cervical cancer a comprehensive review. Int J Gynecol Cancer. 2017;27(1):154-158.

41. Frumovitz M, Ramirez PT, Levenback CF. Lymphatic mapping and sentinel lymph node detection in women with cervical cancer. Gynecol Oncol. 2008;110(3 Suppl 2):S17-S20.

42. Dostalek L, Âvall-Lundqvist E, Creutzberg C, et al. ESGO survey on current practice in the management of cervical cancer. Int $J$ Gynecol Cancer. 2018;28(6):1226-1231.

43. Buda A, Papadia A, Di-Martino G, et al. Real-time fluorescent sentinel lymph node mapping with indocyanine green in women with previous conization undergoing laparoscopic surgery for early invasive cervical cancer: comparison with radiotracer \pm blue dye. J Minim Invasive Gynecol. 2018;25(3):455-460

44. Siesto G, Romano F, Fiamengo B, et al. Sentinel node mapping using indocyanine green and near-infrared fluorescence imaging technology for uterine malignancies: preliminary experience with the da vinci $x i$ system. J Minim Invasive Gynecol. 2016;23(4):470-471.

45. Kadkhodayan S, Hasanzadeh M, Treglia G, et al. Sentinel node biopsy for lymph nodal staging of uterine cervix cancer: a systematic review and meta-analysis of the pertinent literature. Eur J Surg Oncol. 2015;41(1):120 .

46. Mayoral M, Paredes P, Domènech B, et al. 18F-FDG PET/CT and sentinel lymph node biopsy in the staging of patients with cervical and endometrial cancer. Role of dual-time-point imaging. Rev Esp Med Nucl Imagen Mol. 2017;36(1):20-26.

47. Jimenez-Heffernan A, Ellmann A, Naoki-Sado H, et al. Results of a prospective multicenter international atomic energy agency sentinel node trial on the value of SPECT/CT over planar imaging in various malignancies. J Nucl Med. 2015;56(9):1338-1344.

48. Wang L, Liu F. Meta-analysis of laparoscopy sentinel lymph node mapping in endometrial cancer. Arch Gynecol Obstet. 2018;298(3):505-510.

49. Schiavone MB, Zivanovic O, Zhou Q, et al. Survival of patients with uterine carcinosarcoma undergoing sentinel lymph node mapping. Ann Surg Oncol. 2016;23(1):196-202.

50. Schiavone MB, Scelzo C, Straight C, et al. Survival of patients with serous uterine carcinoma undergoing sentinel lymph node mapping. Ann Surg Oncol. 2017;24(7):1965-1971.

51. Rossi EC, Kowalski LD, Scalici J, et al. A comparison of sentinel lymph node biopsy to lymphadenectomy for endometrial cancer staging (FIRES trial): a multicentre, prospective, cohort study. Lancet Oncol. 2017;18(3):384-392.
52. Smith AJ, Fader AJ, Tanner EJ. Sentinel lymph node assessment in endometrial cancer: a systematic review and meta-analysis. Am J Obstet Gynecol. 2017;216(5):459-476.

53. Papadia A, Imboden S, Siegenthaler F, et al. Laparoscopic indocyanine green sentinel lymph node mapping in endometrial cancer. Ann Surg Oncol. 2016;23(7):2206-2211.

54. Paley PJ, Veljovich DS, Press JZ, et al. A prospective investigation of fluorescence imaging to detect sentinel lymph nodes at robotic-assisted endometrial cancer staging. Am J Obstet Gynecol. 2016;215(1):117.e1-7.

55. Buda A, Crivellaro C, Elisei $\mathrm{F}$, et al. Impact of indocyanine green for sentinel lymph node mapping in early stage endometrial and cervical cancer: comparison with conventional radiotracer $(99 \mathrm{~m}) \mathrm{Tc}$ and/ or blue dye. Ann Surg Oncol. 2016;23(7):2183-2191.

56. Niikura H, Kaiho-Sakuma M, Tokunaga H, et al. Tracer injection sites and combinations for sentinel lymph node detection in patients with endometrial cancer. Gynecol Oncol. 2013;131(2):299-303.

57. Rossi EC, Jackson A, Ivanova A, et al. Detection of sentinel nodes for endometrial cancer with robotic assisted fluorescence imaging: cervical versus hysteroscopic injection. Int $J$ Gynecol Cancer. 2013;23(9):1704-1711

58. Buda A, Passoni P, Corrado G, et al. Near-infrared fluorescenceguided sentinel node mapping of the ovary with indocyanine green in a minimally invasive setting: a feasible study. J Minim Invasive Gynecol. 2017;24(1):165-170.

59. McCann GA, Cohn DE, Jewell EL, et al. Lymphatic mapping and sentinel lymph node dissection compared to complete lymphadenectomy in the management of early-stage vulvar cancer: A cost-utility analysis. Gynecol Oncol. 2015;136(2):300-304.

60. Van der Vorst JR, Hutteman M, Gaarenstroom KN, et al. Optimization of near-infrared fluorescent sentinel lymph node mapping in cervical cancer patients. Int J Gynecol Cancer. 2011;21(8):1472-1478.

61. Brar H, Hogen L, Covens A. Cost-effectiveness of sentinel node biopsy and pathological ultrastaging in patients with early-stage cervical cancer. Cancer. 2017;123(10):1751-1759.

62. Schwendinger V, Müller-Holzner E, Zeimet AG, et al. 2006. Sentinel node detection with the blue dye technique in early cervical cancer. Eur J Gynaecol Oncol. 2006;27(4):359-362. 